cemoti $\begin{aligned} & \text { Cahiers d'études sur la Méditerranée } \\ & \text { orientale et le monde turco-iranien }\end{aligned}$

26 | 1998

L'individu en Turquie et en Iran

\title{
Le drapeau turc, emblème de la nation ou signe politique?
}

Claire MAUSS-COPEAUX et Etienne COPEAUX

\section{(2) OpenEdition \\ 12 Journals}

Édition électronique

URL : http://journals.openedition.org/cemoti/633

DOI : $10.4000 /$ cemoti.633

ISSN : $1777-5396$

Éditeur

AFEMOTI

Édition imprimée

Date de publication : 1 juin 1998

ISSN : 0764-9878

Référence électronique

Claire MAUSS-COPEAUX et Etienne COPEAUX, « Le drapeau turc, emblème de la nation ou signe politique? ", Cahiers d'études sur la Méditerranée orientale et le monde turco-iranien [En ligne], 26 | 1998, mis en ligne le, consulté le 08 septembre 2020. URL : http://journals.openedition.org/cemoti/633 ; DOI : https://doi.org/10.4000/cemoti.633

Ce document a été généré automatiquement le 8 septembre 2020.

Tous droits réservés 


\title{
Le drapeau turc, emblème de la nation ou signe politique?
}

\author{
Claire MAUSS-COPEAUX et Etienne COPEAUX
}

Ce début de recherche sur les symboles de la nation en Turquie prolonge des travaux antérieurs sur la mémoire collective et sur l'image en tant que récit iconographique (cartes analysées en tant qu'images, photographies d'événements historiques : Copeaux, 1994, et Mauss-Copeaux, 1996). Notre propos est d'examiner ici l'usage social du drapeau national dans la Turquie d'aujourd'hui où sa fréquence d'apparition dans la vie publique et même privée frappe très vite l'observateur extérieur. C'est d'autant plus remarquable que la loi du 29 mai 1936 restreint son usage à des circonstances précises : "Le drapeau ne peut être déployé sur les maisons et les boutiques que lors des fêtes nationales ". Notre première approche de ce phénomène de pavoisement permanent et quasi généralisé consiste en une analyse de l'apparition du drapeau dans la presse. Notre recherche rejoint celle de Michael Billig (1997, 36-59), qui distingue deux états dans l'apparition publique de l'emblême de la nation : d'une part, the waved flag, expression que nous rendrons par la notion de drapeau-objet : il flotte au vent, représente la nation, doit être salué par les militaires et ne peut souffrir, conformément aux rituels de notre époque, aucune atteinte physique ou même verbale; d'autre part, the unwaved flag, qui correspond partiellement à ce que nous appellerons ici le drapeau-image, tel qu'il est photographié - à dessein ou par hasard dans la presse, tel qu'il est représenté dans les manuels scolaires ou tel qu'il est apposé sous forme de vignette sur les automobiles ou sur tout autre objet; dans ce second état, le drapeau ne requiert pas le même respect : on peut jeter à la poubelle une page de journal sur laquelle il se trouve figuré. L'objet de notre analyse est l'usage de cet unwaved flag, de ce drapeau-image, en Turquie; il s'agit d'images innombrables qui ne sont pas consciemment perçues par le public mais qui contribuent à entretenir, selon Michael Billig et conformément à nos propres observations, un état d'esprit nationaliste.

Le corpus sur lequel nous avons travaillé est formé de plusieurs centaines de photographies de presse couvrant l'année 1996, retenues selon un seul critère : la présence du drapeau turc dans le champ de l'image. Elles proviennent d'un 
dépouillement régulier de sept quotidiens auquel viennent s'ajouter épisodiquement d'autres apports de journaux d'opinion ou destinés au grand public. Notre analyse a porté sur les images elles-mêmes, mais aussi sur leur rapport avec leur co-texte ; nous avons emprunté à l'analyse de discours la notion de co-occurrence pour désigner les cas d'apparition conjointe de deux éléments sur un même cliché (image de drapeau et image de douleur par exemple). De même, parlerons-nous de co-occurrence pour désigner l'apparition conjointe d'un élément du discours photographique et d'un élément d'un discours textuel.

Pour bien situer les événements analysés, il est nécessaire de se remémorer le contexte de l'année 1996 ; le 24 décembre 1995, les élections générales anticipées avaient donné une courte majorité au Parti de la prospérité (Refah, islamiste) dirigé par Necmettin Erbakan. Les efforts des politiciens ont réussi, durant les six premiers mois de l'année, à maintenir ce courant majoritaire à l'écart du pouvoir. Mais, au début de juillet, un gouvernement de coalition dirigé par Necmettin Erbakan, en association avec Tansu Çiller (Parti de la juste voie, DYP) obtenait la confiance du Parlement. Alors que s'accomplissait ce processus la Turquie entrait dans une union douanière avec l'Europe

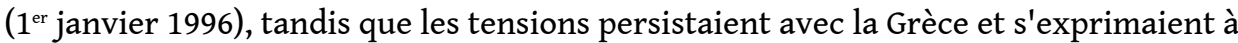
propos de la souveraineté sur Kardak (Imia), un îlot inhabité situé à quelques kilomètres de la côte turque ; en août, deux crises dramatiques éclataient à Chypre, donnant l'occasion à la presse de raviver les réflexes nationalistes; enfin, durant toute l'année, comme toujours depuis 1984, la guerre dans le sud-est de la Turquie continuait ses ravages sans qu'une solution politique n'apparaisse.

\section{6, Une année sous le signe du drapeau}

L'examen des photographies collectées fait apparaître l'existence de rituels, le plus souvent étatiques ou para-étatiques, qui trouvent leur sens dans un certain savoir et dans un rapport au sacré, que nous évoquerons plus loin. Certains de ces rituels sont accomplis selon une " récurrence de l'identique", tandis que d'autres sont convoqués ou apparaissent en liaison avec des circonstances particulières (Abélès, 1990).

Le drapeau au quotidien : un tissu de signes

La fonction phatique des images

L'une des difficultés de l'analyse réside en l'existence d'une multitude de photographies difficiles à classer ; elles sont banales, répétitives, n'illustrent aucun événement notable ou mémorable, mais sont du ressort de la vie sociale quotidienne, des petites fêtes, célébrations, rencontres insignifiantes. Ces drapeaux-images, les unwaved flags, correspondent à la simple présence du drapeau-objet dans le paysage quotidien, la photographie n'étant ici que le reflet d'une certaine vie sociale. Le drapeau n'est photographié que parce qu'il est, presque inévitablement, dans le champ de l'image, tout en étant hors-sujet. Si les notions de champ et de hors champ ne peuvent prêter à confusion, celles de sujet et hors sujet, appliquées à l'analyse d'une image, nécessitent une première remarque méthodologique. Nous dirons que le drapeau est sujet s'il est photographié à dessein ; il sera hors sujet s'il ne figure sur l'image que parce qu'il était par hasard dans le champ de l'opérateur à l'instant du cliché ; mais peut-on jamais invoquer avec certitude le hasard et l'absence d'intention pour analyser une photographie?

Il existe au moins deux moyens de déterminer si le drapeau qui figure dans le champ d'une photographie est sujet ou hors sujet ; le premier est l'examen du co-texte - la légende de la photographie ou l'article qu'elle illustre ; si celui-ci mentionne, rappelle, souligne, commente la présence du drapeau dans le champ de l'objectif, on peut le 
considérer comme sujet de l'image ; dans ce cas le drapeau parle; dans le cas contraire, il est hors-sujet et nous qualifierons sa présence de muette. Le second moyen est de distinguer les photographies sur lesquelles le sujet est étroitement cadré, de celles dont le sujet semble "flotter" dans un cadre trop large pour lui. Dans le premier cas, le drapeau, placé en arrière-plan, du sujet peut être photographié par hasard et est donc hors-sujet; dans le second, il apparaît souvent que le photographe a élargi, parfois considérablement, le champ de la prise de vue de manière à y comprendre le drapeau ; le sujet (portrait d'un orateur par exemple) est alors connoté par la présence du drapeau qui devient un second sujet de la photographie ${ }^{1 .}$

Bien que les images répondant au critère du drapeau hors-sujet soient infiniment variées, on peut en distinguer deux types. Le premier consiste en photographies protocolaires, notamment celles qui représentent le président de la République ou un haut personnage de l'État dans l'exercice de ses fonctions (inaugurations, discours), les autorités de l'État, comme lors des réunions du Conseil national de sécurité (MGK) ou de la Haute assemblée militaire (YAS) ; le drapeau étant un attribut conventionnel de l'autorité politique, les dignitaires peuvent difficilement être cadrés sans qu'il n'apparaisse à l'arrière-plan ; toutefois, comme le montrent bien des exemples, la chose est possible. Le second type rassemble des photographies très banales rendant compte de séances publiques, assemblées, colloques, commémorations, inaugurations, fêtes, au cours lesquelles le drapeau est accroché à la tribune des orateurs, ou posé au mur du fond derrière la tribune, de sorte qu'il est difficile également de cadrer les orateurs sans l'inclure dans le champ.

Néanmoins, il serait trop facile d'attribuer au hasard sa présence dans le champ de l'objectif ; le photographe peut cadrer sa composition sans intention consciente, mais en résultat d'un habitus patriotique (Veyne, 1974) et afin que le drapeau donne du sens à la photographie. En effet, la photographie de presse, telle que la déchiffre lecteur, résulte de multiples choix : ceux du photographe, qui travaille apparemment en toute liberté, mais dont le regard est en réalité guidé par une éducation visuelle et des valeurs de référence, les stéréotypes photographiques existant - les "clichés" - et la nécessité de se conformer aux conventions pour plaire ou pour vendre : en somme, la prise de vue résulte, consciemment ou non, des modes iconographiques ambiantes et des demandes du marché de l'image ; elle résulte également des choix des metteurs en page qui choisissent les clichés en rapport avec l'article, et qui peuvent éventuellement les recadrer; des choix inconscients du lecteur, dont le regard peut s'arrêter ou passer sur un cliché, mais dont le simple fait qu'il feuillette ce journal provoque des impressions qui restent à l'état latent dans la mémoire ; enfin, on ne peut écarter une éventuelle intervention du sujet lui-même sur le cadrage : par exemple, il nous est arrivé qu'une personne nous demande de la photographier avec le drapeau turc en arrière-plan; l'intention du sujet répond là aussi à une conformation inconsciente à un stéréotype.

Face à cette partie du corpus qui regroupe les photographies anodines, il convient de réfléchir à la présence si fréquente du drapeau dans tant de circonstances de la vie publique ou même privée, comme certains mariages. En linguistique, on qualifie de phatiques les paroles insignifiantes qui sont prononcées uniquement dans le but de maintenir le contact entre le destinateur et le destinataire. Nous proposons de qualifier également de phatique cette présence quasi permanente du drapeau dans la vie turque et, par contre-coup, dans le champ de l'objectif des photographes de presse. Le drapeau, objet phatique, maintient en permanence un double contact : la nation affiche 
sa présence ; le citoyen qui pavoise exprime son adhésion, manifeste sa fierté, revendique son appartenance.

Les rituels codifiés

Dans d'autres cas, facilement identifiables par leur répétitivité même, le drapeau-image est l'élément d'un rituel ; il s'agit bien d'une " récurrence de l'identique ". En 1996, il en était ainsi d'une bonne partie des obsèques relatées par la presse nationale et de nombreuses photographies d'arrestations; dans les deux cas, nous avons affaire à des rituels dramatisés.

La loi de mai 1936 évoquée ci-dessus permet de revêtir du drapeau national les cercueils des martyrs (Òehit) ${ }^{2}$, ceux des Turcs morts à l'étranger alors qu'ils y occupaient une fonction officielle, et ceux des citoyens qui ont rendu des services insignes à la nation. Alors que le cercueil d'un musulman ordinaire est revêtu d'un poêle vert brodé de versets du Coran, dans les cas énumérés ci-dessus, au cours de la cérémonie, la famille ou les proches étendent sur ce poêle le drapeau au croissant et à l'étoile ; c'est l'acte dit sarýlma.

Lors de la prise de vue, le cercueil est généralement placé entre la famille, ou l'officiant, et le photographe. Aussi, barre-t-il l'image d'un rectangle rouge ; sur la photographie elle-même, le co-occurrent iconique du drapeau est une image de la douleur (représentée par un ou plusieurs membres de la famille étreignant le cercueil - et le drapeau) ou de la gravité (l'officiant, et, s'il s'agit des obsèques d'un soldat, les officiels, les militaires assistant à la cérémonie, les nöbetçi ou militaires de faction qui veillent le cercueil). Ici, le drapeau parle. Il est sujet de la photographie, car il en est presque toujours fait mention, dans l'article qui relate la cérémonie, de l'acte du sar_lma. Dans le cas d'un martyr tué au combat, l'image du drapeau s'accompagne souvent de cooccurrents textuels comme hain (traitre) et intikam (vengeance). La présence du drapeau sur le cercueil (drapeau-objet) et sur la photographie (drapeau-image) a une fonction facilement identifiable : il est la métaphore de la gratitude de la nation vis-àvis du martyre, et celle du devoir de vengeance à l'encontre du traître.

Sur les images d'enterrements, le photographe, en privilégiant l'un ou l'autre des acteurs de cette mise en scène dramatique et en répondant à une certaine recherche esthétique, est autorisé à exprimer à la fois son sentiment et sa culture. Les photographies d'un autre type de rituel, l'arrestation, sont encore plus rigoureusement codifiées. Elles se rapprochent, dans leur forme et leur rigidité, des photographies judiciaires. Lors de l'arrestation, le cérémonial est imposé par les policiers. La presse est convoquée dans les locaux de la Sûreté, du commissariat ou de la gendarmerie. Généralement, un symbole du pouvoir est disposé sur le mur qui va servir de fond à la photographie : drapeau, buste d'Atatürk, carte de la Turquie ; leur signification est équivalente : manifestation d'une polysémie du signifié-nation. Nous envisageons plus loin des manifestations de cette polysémie du signifié dans la littérature scolaire. Le délinquant, ou le groupe de délinquants, est placé entre le drapeau et une table sur laquelle sont déposées les pièces à conviction. S'il y a des armes, les balles sont disposées de manière à former le sigle de l'équipe policière qui a procédé à l'arrestation. Parfois, s'il s'agit de militants d'extrême-gauche ou kurdes, l'emblême du mouvement répond en écho dérisoire au drapeau disposé en arrière-plan ${ }^{3}$. L'attitude des suspects est variable : celle des petits truands est généralement contrite; les militants politiques ont souvent des regards de défi et quelques-uns lèvent le poing ou font le signe de la victoire ; mais, parfois, l'ensemble des suspects tourne le dos à l'objectif et s'incline devant le drapeau. 
Le drapeau, ici, est muet : l'article ne signale jamais sa présence ; toutefois, on est tenté de dire qu'il n'est pas hors sujet, tant sa présence est signifiante dans le rituel photographié. Il est l'ordre devant lequel s'inclinent, parfois physiquement, les appréhendés. Il est, d'ailleurs, seul représentant de l'ordre, car la police reste toujours hors champ.

Les récurrences de ces clichés stéréotypés (obsèques, arrestations), dont la composition même répond à des standards, sont fréquentes, quasi quotidiennes. Avec les apparitions phatiques du drapeau, cet ensemble forme lui-même un rituel journalistique, un peu comme le sont les monotones photographies de dépôts de gerbes aux monument aux morts dans la presse régionale française ; mais, alors qu'en France le rituel n'apparaît qu'à des moments précis du cérémonial républicain (notamment le 11 novembre), en Turquie, il est étalé dans le temps, de manière à former un tissu de signes sous-jacent aux crises et aux commémorations particulières : entre les moments d'expression paroxystique du patriotisme, le drapeau reste présent dans la presse, se rappelle sans cesse à la mémoire du lecteur-citoyen, est rappellé par son occurrrence sa fonction de rassembleur, de garant de l'ordre (photos d'arrestations), de symbole patriotique (commémorations), quel que soit le prix de la fidélité qu'il requiert (enterrements).

Le drapeau en crises

Les crises sont des événements séparés du quotidien, mais elles apparaissent dans le contexte du tissu de signes défini plus haut; elles ne sont qu'épisodes plus denses, paroxystiques, de l'apparition quasi quotidienne, phatique, du drapeau, de sorte qu'il est légitime de se demander si le tissu de signes n'assure pas une fonction de relais entre les crises, et même s'il ne nourrit pas, à la limite, l'éclosion de crises liées au drapeau. Sur les images de crise, et même dans son déroulement réel, le drapeau est sujet actant : c'est lui qui provoque, focalise, détermine la crise. Puis, une fois celle-ci enclenchée ou passée, une image photographique en est retenue, selon un processus qui reste à étudier, par l'ensemble de la presse; tel un logo, cette image devient par la suite l'identificateur de l'événement et réapparaît lorsqu'il en est à nouveau question dans les journaux.

Il ne s'agit pas ici de faire le récit des crises de 1996 : nous nous contentons de les replacer dans le contexte sémiologique défini ci-dessus.

La crise de Kardak (27 janvier - 1 février 1996)

Cette crise fut relative à un îlot dont la souveraineté est contestée; elle a éclaté dans un contexte de tension gréco-turque durable en mer Égée, l'image de la Grèce étant, dans le discours historique officiel ainsi que dans la presse, très négative. La crise, immédiatement qualifiée de " guerre du drapeau " par la presse (Hürriyet, 28 janvier 1996), a été provoquée par l'implantation, sur l'îlot, d'un drapeau grec, aussitôt retiré par des journalistes zélés de Hürriyet, et remplacé par un drapeau turc. À en croire les manchettes des jours suivants, l'enjeu n'était plus la possession de l'îlot mais la présence du drapeau qui en symbolise la possession, comme si l'apposition du signe pouvait régler le problème de la souveraineté mieux que ne le ferait une négociation entre les deux Etats.

L'élément le plus intéressant de cette affaire est son origine journalistique. L'initiative des journalistes de Hürriyet répondait à l'habitus patriotique forgé par l'éducation, l'armée, la propagande, et, en dernier ressort, l'État. Il est difficile, pour les représentants d'un État qui incite aux manifestations extérieures de patriotisme souvent sous des formes affectives -, de désavouer les initiatives des citoyens zélés, 
surtout lorsqu'elles ont déjà trouvé un vif écho dans la presse et l'opinion publique. Aussi, l'ampleur de la réaction a forcé l'État et même l'armée à intervenir ; cette dernière ne peut sembler indifférente aux questions de drapeau alors qu'elle est la gardienne des symboles : l'État est alors piégé dans une logique de surenchère par des citoyens qui ont pris son enseignement au pied de la lettre. Nous retrouverons d'ailleurs le même processus dans d'autres circonstances. La crise a produit son image identificatrice : la photographie des journalistes de Hürriyet, décoiffés par le vent, plantant le drapeau turc sur l'îlot, avec, en arrière-plan, l'hélicoptère de l'expédition ${ }^{4}$; mais un autre type de cliché, qui pourtant ne comporte pas l'image du drapeau dans son champ, a été lui aussi utilisé ; il représente un commando de marine, formation que la crise a popularisée, partant à l'assaut en canot pneumatique. C'est cette image de commando qui a été superposée à celle du drapeau par Hürriyet pour composer son extraordinaire première page du 30 août 1996 (jour de la fête de la Victoire) ; il ne s'agit plus en fait d'un unwaved flag, drapeau-image, mais bien d'un waved flag, drapeauobjet couvrant toute la page ${ }^{5}$, qu'il aurait peut-être été délicat de jeter à la poubelle devant témoins.

Durant les mois qui ont suivi la crise de Kardak, l'état des relations gréco-turques n'a cessé d'être débattu dans la presse. Par ailleurs, la guerre dans le sud-est a continué ses ravages, et le 9 avril 1996 la Turquie a été stupéfaite par l'ampleur des pertes de l'armée turque (27 morts) au cours d'un accrochage avec le PKK. Le premier mai et ses violences (3 morts) ont encore secoué le pays ; dans tous les cas, la ferveur patriotique a été relancée : après une " menace " extérieure (Kardak), l'opinion a vu réapparaître les menaces séparatiste et d'extrême-gauche ; les photographies des défilés du premier mai, violemment colorés en rouge (kýzýl) par les drapeaux et uniformes des mouvements révolutionnaires, ont fait écho aux drapeaux nationaux, rouges également (al) arborés lors des enterrements de martyrs ${ }^{6}$. L'extrême-gauche légale elle-même s'est jointe aux manifestations de patriotisme : "Lors de la guerre de Libération [1922], ceux qui s'en sont pris au drapeau turc et qui voulaient ruiner la Turquie ont été jetés dans la mer Égée ; ceux qui s'en prennent à ce même drapeau seront eux aussi rejetés dans n'importe quelle mer." (Do_u Perinçek, président du Parti des travailleurs, cité par Ayhan Songar dans Türkiye le 5 mai 1996).

Le congrès du HADEP (23-29 juin 1996)

À la fin du mois de juin 1996, alors que la conférence mondiale de l'ONU Habitat II s'était déroulée sans accroc à Istanbul, la Turquie a été à nouveau secouée par une affaire de drapeau. Lors du deuxième congrès du HADEP (Halkýn Demokrat Partisi, parti pro-kurde légal), à Ankara, des provocateurs firent tomber l'immense drapeau turc qui ornait la salle et le remplacèrent par la bannière du PKK et le portrait d'Abdullah Öcalan, son dirigeant (23 juin 1996). C'est une nouvelle fois Hürriyet qui contribua à mettre le feu aux poudres en présentant en première page, dès le 24 juin, l'instantané de la chute du drapeau turc ; deux jours plus tard, cette photo désormais célèbre a été publiée dans tous les quotidiens ; à la télévision, la scène de la chute du drapeau a été diffusée des dizaines de fois. Cette photo devient le logo de l'événement ; elle évoque l'offense à la nation, le danger de déstabilisation ou de sécession, en somme, tout ce qui menace la Turquie d'aujourd'hui. À partir du 25 juin apparut le mot qui devint le co-occurrent fréquent de l'image de la chute du drapeau : " traître " (hain). La photo, et le mot, firent des apparitions régulières au moins jusqu'au 28 août, date de reconstitution du délit après l'arrestation du coupable présumé, Faisal Akcan. 
Autant que l'événement, la réaction “ populaire ” qui s'ensuivit est intéressante. Lancée à l'origine par l'extrême-droite (Parti de la grande union, BBP puis Parti nationaliste du mouvement, MHP), l'opération " Pavoisez ! " "Bayrak as !") a été très vite officialisée (circulaire du ministère de l'Éducation, puis d'autres services officiels) et généralisée. Présentée comme une réaction spontanée de la population, les résultats de l'opération firent très vite la première page des journaux et renforcèrent encore la campagne de pavoisement. Deux types de clichés ont été retenus par les journaux pour symboliser la réaction ; l'un, qui représente une importante galerie du grand bazar d'Istanbul pavoisée de rouge et blanc, était déjà un stéréotype utilisé à l'occasion des grandes commémorations (cf. Türkiye, 29 octobre 1995); il symbolise l'unanimité nationale dans un cadre historique, populaire, reconnu à l'étranger ; l'autre est une série de clichés d'une manifestation de protestation contre le PKK, à Erzurum, due à l'initiative d'un religieux respecté, Naim Hoca, et des esnaf, les corporations d'artisans et commerçants. Ces images d'Erzurum semblent avoir été choisies pour représenter l'Anatolie lointaine, celle qui souffre de la guerre (des villages proches d'Erzurum avaient été attaqués par le PKK quelques jours plus tôt) et qui reste néanmoins fidèle à la patrie. Les deux stéréotypes ont un sens très net de représentation d'une nation qui se dresse et fait obstacle à la division. Il faut noter encore que la campagne "Pavoisez!" a densifié l'apparition phatique du drapeau : par exemple, nombre de taxis arboraient encore, au début de 1997, un drapeau-vignette sur leur capot. Les objets décorés du drapeau national se sont mis à pulluler : insignes, vaisselle, cartes postales, bonnets, tee-shirts, porte-clés, vignettes, briquets, etc.

Les incidents de Chypre (11- 14 août 1996)

Le drapeau turc est resté densément présent dans les journaux durant les mois de juillet et d'août, mais sans que ne se dégage aucun stéréotype. Les événements heureux et malheureux se sont succédés : les Jeux olympiques d'Atlanta, avec leurs succès turcs (19-26 juillet), représentent l'avers de la crise du HADEP ; l'apparition du drapeau dans la presse de ces semaines exprimait la joie, la fierté ; le héros haltérophile Naim Süleymano_lu l'emporte alors sur le " traitre " Faisal Akcan, suspecté de la provocation du 23 juin. Mais au même moment, la Turquie est secouée par une meutrière grève de la faim dans les prisons ; des manifestations de soutien, notamment dans le quartier alevi de Gaziosmanpaòa à Istanbul, ont pris un tour très violent et ont suscité leur répression par les forces de l'ordre. La presse a montré des drapeaux turcs lacérés par les manifestants, et les drapeaux rouges (k_z_l) de ces derniers ont à leur tour investi les pages des journaux ; la reprise de contrôle du quartier par la police a été signifiée par une nouvelle image en contre-plongée symbolisant la reconquête : un policier, nouvel avatar d'Ulutbatl_ Hasan, plantant triomphalement le drapeau turc au sommet d'un bâtiment important du quartier, accomplit ainsi un geste par lequel les autorités manifestent qu'elles situent l'extrême-gauche hors de la collectivité nationale ${ }^{7}$. Le mois de juillet est suffisamment empreint de ferveur pavoisante pour qu' $^{\prime} \mid \mathrm{brahim}$ Tatl_ses, lors de son tour de chant à Diyarbak_r (considérée comme la capitale du Kurdistan par les militants kurdes), arbore le drapeau national sur scène. Juillet est aussi le temps de la commémoration de l'invasion de la partie nord de Chypre par l'armée turque (1974), marquée par une visite du nouveau premier ministre islamiste, Necmettin Erbakan, à Nicosie.

C'est donc dans une atmosphère empreinte de patriotisme qu'éclatent les crises de Chypre (11 et 14 août 1996), où tout incident est plus dangereux, car les manifestations d'orde idéologie, des deux côtés de la zone-tampon de l'ONU qui partage l'île, y est plus 
marquée, et parce que Chypre est un territoire de référence des deux nationalismes opposés, turc et grec. Tous les événements qui précèdent, et surtout leurs commentaires dans la presse, ont joué leur rôle dans l'exaspération du sentiment national. Le 11 août, lors d'une manifestation dans la zone-tampon, un Chypriote grec avait été battu à mort. Le 14, après ses funérailles, un Chypriote grec qui tentait de monter à la hampe d'un drapeau turc pour le décrocher, fut tué par balles. Il n'avait qu'une bien faible chance d'y parvenir : la hampe mesurait douze mètres et il en avait gravi moins de trois. Pourtant des Chypriotes turcs ont tiré, et le provocateur est mort ${ }^{8}$. L'événement est typique à deux égards ; la culture patriotique avait tellement sacralisé le drapeau et induit des pensées-réflexes qu'on a eu le réflexe de tirer et de tuer, sans chercher un autre moyen d'empêcher l'incident. Consciemment ou non, le ou les auteurs des coups de feu se sont sentis légitimés par l'amalgame créé entre l'offense au drapeau et la traîtrise. Du reste, les réactions des responsables turcs, et notamment celle de Tansu Çiller, ont légitimé a posteriori le meurtre du Chypriote, comme nous le verrons plus loin.

Comme pour les événements antérieurs, ceux de Chypre ont produit des clichés stéréotypés, qui ont servi ensuite d'identificateurs. La photo du Chypriote grec grimpant à la hampe du drapeau turc, acte dérisoire qui a cependant provoqué sa mort, a été reproduite des dizaines de fois dans tous les journaux. Le drapeau turc en est le sujet mais il est hors champ, restant dans l'implicite. Dans le champ, par contre, au premier plan, figurent des casques bleus qui regardent passivement la scène. La photographie, ici, est devenue le stéréotype de la menace grecque, mais aussi de la passivité - selon la presse turque - de l'ONU.

L'été, puis l'année 1996, se sont terminés sans autre incident grave ${ }^{9}$, mais trois célébrations nationales ont permis de nouveaux pavoisements : la fête de la victoire (30 août), la fête de la République (29 octobre) et l'anniversaire de la mort d'Atatürk (10 novembre). En somme, au flot quasi quotidien des représentations du drapeau et aux photographies des rituels ordinaires de l'État turc (protocole, obsèques, arrestations) se sont ajoutés des stéréotypes nés des images de crises : le drapeau turc sur l'îlot de Kardak, la chute du drapeau lors du congrès du HADEP et les images du pavoisement consécutif (celles du grand bazar et celles des manifestants d'Erzurum), le Chypriote grimpant au mât. Certains de ces stéréotypes ont même servi à construire d'autres images, des caricatures par exemple (Zaman, 4 février ; Cumhuriyet, 16 août).

\section{La polyvalence des signes}

Les images de la presse, éléments d'une chaîne d'occurrences

Les apparitions du drapeau dans les journaux forment un discours qu'on peut et doit rapprocher d'autres discours, prononcés en d'autres lieux et dans un autre langage. Le discours photographique de la presse est continuation, répétition et rappel permanents de l'habitus patriotique formé à l'école ; il renvoie aussi, plus généralement, à un ensemble d'habitudes relatives aux couleurs nationales, ce qui rend nécessaire une interrogation plus générale sur la signification de la couleur rouge en Turquie. Les photographies des quotidiens répondent à deux types de stéréotypes, au moins. Dans la dimension spatiale, elles renvoient aux autres occurrences simultanées du drapeau - ou de la couleur rouge dans certains cas - dans le quotidien même : beaucoup de journaux ont un logo comportant un drapeau (c'est le cas par exemple de Hürriyet, Türkiye, AkÒam, Önce Vatan, Gözcü, Ortadogu, Posta, Fanatik) ; la plupart sont de couleur rouge, qui est aussi la couleur dominante de la page des sports, même si le drapeau n'y apparaît pas si fréquemment. De même, dans la publicité, l'utilisation d'un 
fond rouge, sur lequel se détache en blanc un slogan, est trop systématique pour qu'il s'agisse d'un hasard. Coca-Cola et Johnnie Walker profitent d'ailleurs de l'occasion ; d'autres firmes recourent à des mises en scène explicites (Isuzu, Kale) ${ }^{10}$. Enfin, nous disposons d'un exemple typique de l'usage de la couleur rouge par les metteurs en page avec la page trois du numéro du 30 septembre 1996 de Hürriyet : en haut à gauche apparaît le logo (blanc sur fond rouge) du quotidien ; en haut à droite, une image illustrant un règlement de compte entre truands cadre une large flaque de sang, rouge ; le centre de la page est occupé par la photo d'identité d'un chauffeur de taxi assassiné, sur fond rouge comme c'est l'habitude en Turquie ; en bas à droite, l'illustration d'un reportage sur un mariage collectif montre les robes blanches des fiancées ceintes, comme le veut la tradition, d'un tissu rouge ; la cérémonie est pavoisée de drapeaux turcs, rouges et blancs, et le maire d'Istanbul, qui officie, est couvert d'un vêtement rouge ; enfin, en bas de page, deux annonces publicitaires utilisent la couleur rouge ${ }^{11}$. Dans la dimension temporelle, les occurrences sont redondantes et renvoient les unes aux autres, notamment à l'éducation et à la vie scolaire. Dans toutes les écoles turques, une cérémonie au drapeau a lieu au début de chaque semaine. Les salles de classe sont pavoisées et chacune a son " coin d'Atatürk " (Atatürk köÒesi) dont le soin est confié par roulement hebdomadaire à des élèves de service (nöbetçi). Ce cérémonial paramilitaire est à son tour représenté " en abyme " dans les manuels de lecture, les abécédaires, qui multiplient les images représentant l'école, comportant évidemment des drapeaux, des représentations de cérémonies patrioques (cérémonies à Atatürk, défilés d'enfants, enfants assistant à des défilés militaires, etc.). De plus, les images comportant des drapeaux deviennent plus nombreuses au cours des années ; en 1990, le manuel de langue turque pour écoles primaires premier niveau (Éditions de l'Éducation nationale) comportait vingt-quatre représentations de drapeaux et trente représentations de l'un des symboles patriotiques que sont le drapeau, le militaire ou la carte de la Turquie. En 1993, ces chiffres étaient passé respectivement à trente-deux et quarante-et-un.

Le discours iconographique a tendance à fondre le drapeau avec les autres signes, notamment la carte du pays et le portrait d'Atatürk. On voit apparaître vers 1990 un modèle de carte-drapeau qui a été largement diffusé depuis ; parfois, le portrait d'Atatürk y est ajouté (Kantik, 46 ; Copeaux, 1998). Un simple coup d'œil aux petits carnets vendus aux militaires dans les villes de garnison, dans les années 90, fait découvrir les mêmes tendances : les symboles de l'État, et les connaissances patriotiques minimales requises du soldat, sont les mêmes que celles diffusées à l'école, la caserne apparaissant au moins dans cette perspective le prolongement de celle-ci. Plus profondément, les leçons d'histoire, à différents niveaux de l'enseignement, enracinent dans l'esprit la sacralité du drapeau. C'est dans ces leçons qu'est fabriqué le stéréotype relatif à l'origine de la couleur rouge du drapeau (le sang des martyrs) qu'on voit réapparaître dans les discours accompagnant la crise du congrès du HADEP, et qui est répandu par l'imagerie populaire du parti d'extrême-droite MHP, très diffusée. Les idées de sang (des sacrifiés), répandu sur le sol de la patrie, pour défendre le territoire de la future république kémaliste ont leur répondant imagé dans l'image triple de la carte-drapeau sur laquelle figure le visage d'Atatürk. L'association sang-sacrifice-sol conduit à la sacralisation de chacun des trois éléments, et à celle du drapeau qui les évoque tous ensemble. Dans les manuels scolaires de tous niveaux, des expressions ou des textes entiers viennent expliciter ces idées, notamment dans les introductions, où la fonction patriotique de l'enseignement de l'histoire est exposée, dans les leçons sur 
les batailles de Mantzikert et des Dardanelles, dans les leçons d'atatürkçülük (catéchisme kémaliste qui remplace l'histoire contemporaine), et dans certains textes de lecture pour écoles primaires (Copeaux, 1996 et 1997) ; c'est en ce sens que les rituels au drapeau font référence à un certain savoir, en particulier un savoir historique. Enfin, une succession de commémorations nationales (au moins cinq par an) forme une série de paroxysmes réguliers de manifestations canalisées du patriotisme, alors que les crises mentionnées ci-dessus en sont une forme aléatoire irrégulière et peuvent donner lieu à des débordements. Ainsi, trois rythmes d'apparition du drapeau se superposent : la toile de fond composée d'images phatiques, sur lesquelles s'inscrivent les scansions régulières des fêtes patriotiques, et l'explosion des crises.

Le sacré, ou la confusion entre signifiant et signifié

Le signifiant - le drapeau - est polysémique, et le signifié - la nation - l'est également. Tous ces signes que sont la couleur rouge, le drapeau, la carte, le portrait d'Atatürk, renvoient les uns aux autres, et tous les systèmes de signes sont perméables les uns aux autres : les enfants participent aux défilés ; les images de défilés illustrent leurs livres de lecture (lkokul Türkçe, 91); les chants appris à l'école et à la caserne évoquent le drapeau ; les images de crises et les images de commémorations se répondent (par exemple le 30 août) ; les commémorations, en entretenant un climat émotionnel, peuvent faciliter l'éclosion de crises (comme à Chypre); la presse reflète ce système de signes et le nourrit. Elle influe sur l'école et l'armée, les instituteurs et les officiers étant lecteurs des journaux. La publication dans Zaman, le 22 mai 1996, du poème patriotique de Arif Nihat Asya, Bayrak ("Le drapeau "), est un exemple de la perméabilité entre les différents discours ; ce texte appartient à la littérature scolaire et deux de ses vers ont été reproduits sur une vignette quelquefois apposée sur les automobiles :

\begin{tabular}{|l|l|}
\hline “Dalgalandýgýn yerde ne korku, ne & “ Là où tu flottes il n'y a ni \\
keder, & crainte, ni chagrin, \\
Gölgende bana da, bana da yer & À ton ombre, à moi aussi laisse- \\
ver." & moi une place. ” \\
\hline
\end{tabular}

L'un des éléments les plus importants du processus est la confusion entre le signifiant (le drapeau et les autres symboles) et le signifié (la nation). Ceci n'est évidemment pas propre à la Turquie ; on peut admettre que c'est toujours le cas dans le domaine du sacré, et c'est peut-être même une caractéristique du sacré. Mais la sacralisation du signifiant et la confusion entre signifiant et signifié sont telles qu'elles aboutissent, dans la vie politique, à un processus de récupération-confiscation. Un groupe politique, en s'appropriant le drapeau ou un autre symbole, en les arborant au cours d'une manifestation, considère qu'il donne une légitimité à son action, et il exclut de cette légitimité ceux qui n'y participent pas. Les événements de septembre 1955, qui répondaient à un prétendu attentat dirigé contre la maison d'Atatürk à Salonique, avaient déjà provoqué une campagne " Pavoisez !"; ce mot d'ordre proféré dans les rues de Beyo_lu (Istanbul) par des groupes d'extrémistes (principalement les nationalistes du groupe "Chypre est turque " (Kýbrýs Türktür), avait un but nationaliste (la provocation d'une crise anti-grecque, l'intimidation des minorités non musulmanes) travesti sous un aspect patriotique. Aussi, celui qui n'obtempérait pas pouvait-il être désigné à la vindicte publique comme un opposant au drapeau, donc à la 
patrie, un traître. C'est ainsi qu'apparaît, par ce processus, une co-occurrence entre l'image du drapeau et le mot traître.

En somme, la confusion entre signifiant et signifié entraîne une focalisation quasi religieuse des problèmes sur le drapeau ou sur d'autres symboles. Un problème réel comme celui de la souveraineté sur la mer Égée peut se cristalliser sur le drapeau ; dès lors, l'opinion publique et la presse considèrent que le problème est réglé lorsque la question du drapeau est résolue, tandis que, discrètement, les vraies solutions sont cherchées - sincèrement ou non - au niveau diplomatique. Pour l'opinion publique, le problème s'est exprimé en termes de namus, d'honneur ; inversement, l'offense au drapeau peut elle-même créer un problème, comme lors de la crise du congrès du HADEP, ou de la crise de Chypre, notamment parce que la sacralisation du signe est très forte, parce que les protagonistes connaissent l'importance de l'icône dans le système adverse, et parce que les adversaires savent très bien comment s'atteindre et se faire mal à travers les symboles.

Trois niveaux de confusions

Si la confusion entre signifiant et signifié intervient avant tout au niveau du signe luimême, elle se produit également à un second niveau, celui du discours verbal ; la métaphore peut être prise au mot. La phrase prononcée par Tansu Çiller, ministre des Affaires étrangères et vice-premier ministre, peu après le meurtre du Chypriote grec, “Nous briserons les mains de ceux qui s'approchent du drapeau " (presse turque du 16 août 1996) peut accepter deux sens : la locution "que sa main soit brisée " exprime l'idée de punition, de vengeance, donc, dans le contexte politique de cet énoncé, de sanction légale, de mesures, juridiques ou réglementaires, visant à punir ou à prévenir, par effet dissuasif, l'offense au drapeau. Mais si l'on prend la métaphore à la lettre, on peut se sentir légitimé à “ briser les mains ", c'est-à-dire à utiliser la violence. Un caricaturiste a habilement illustré cet énoncé en représentant une Grèce peuplée d'evzones aux mains bandées, tandis que Tansu Çiller, de l'autre côté de la mer Égée, profère la désormais célèbre phrase (Türkiye, 20 août 1996) ${ }^{12}$. Une telle interprétation de l'énoncé de Çiller est une justification a posteriori du meurtre du Chypriote grec battu à mort par les Loups gris turcs, le 11 août, et de celui du Chypriote qui tentait d'atteindre le drapeau turc le 14 août. Le pouvoir turc, pris au piège de sa propre rhétorique et des symboles qu'il a créés, n'a rien fait pour lever l'ambiguité : aucune enquête, aucune sanction, aucun désaveu publics n'ont été relatés par la presse turque. Depuis, l'expression a fait son chemin : elle est souvent prononcée lors des enterrements de Òehit. La métaphore, prise au mot par une opinion travaillée par les media et par l'habitus patriotique, aurait pu pousser d'autres irresponsables à l'action violente. Certains politiciens, dans ce cas, sont enclins à suivre, car il leur est difficile de rester en deçà du niveau de patriotisme créé et renforcé par le système qu'ils représentent : ils ne peuvent paraître moins patriotes que leurs électeurs.

Enfin, on doit noter que la confusion entre signifiant et signifié influence le domaine du droit, puisque, dans le cas de l'affaire du congrès du HADEP, le procureur général, Nuh Mete Yüksel, a proposé que l'acte du provocateur ne soit pas qualifié de simple délit d'offense au drapeau, mais d'“ acte terroriste aussi grave qu'un assassinat " (Hürriyet, 27 août 1996) ${ }^{13}$. Les juges ont suivi le procureur en prononçant en juin 1997 une peine qui paraît démesurée : vingt-deux ans de prison.

\section{Un début d'évolution}

Le résultat de la confusion des divers niveaux de confusion est que le drapeau et ses couleurs déteignent : sur les emblêmes et bannières de certains partis politiques, qui 
utilisent le rouge et le blanc (Refah, DYP, MHP, extrême-gauche) ; sur la vie sportive dans laquelle très souvent, le drapeau national est associé au fanion du club; enfin, sur la publicité. Le drapeau est, le plus souvent, associé à des idées dramatiques (enterrements, arrestations) : nous sommes dans un pays en guerre, où régnait, durant l'été et l'automne 1996, une atmosphère de “ patrie en danger ". Le seul type d'occurrence où le drapeau ait été associé à l'idée de joie, de victoire, de succès, est le sport, et c'est peut-être l'une des raisons du succès populaire du football, et de la confusion fréquente entre rencontre sportive et manifestation nationaliste : l'actualité, en dehors de ces matches et des commémorations officielles, ne permettait pas autrement d'exulter, d'extérioriser le sentiment patriotique si fortement cultivé en Turquie ; en outre, le football et ses rituels de foule contribuent à entretenir parmi la population masculine jeune l'esprit que l'Etat requiert d'elle en cas d'affrontement. Les réactions prises en compte par la presse dans les jours qui ont suivi la crise du HADEP forment un discours parallèle à celui des images du drapeau, un co-texte, qui présente bien des points communs avec d'autres discours. On peut y distinguer, parmi les co-occurrents, les traîtres et les associés. Les traîtres sont tous ceux qui portent la main sur le drapeau. “ Ils n'ont ni sang ni âme " (kansýz, cans_z), ils n'ont aucun respect pour leurs pères et leurs aïeux. Les associés sont la carte du pays et le portrait d'Atatürk; ils sont interchangeables dans certaines occasions mais non dans toutes. L'atmosphère de " synthèse turco-islamique " ${ }^{14}$ qui prévaut en Turquie fait que les mosquées sont, elles aussi, souvent pavoisées du drapeau, à l'exclusion de tout autre symbole national. D'autre part, la religion est associée au symbole national dans certains slogans fréquemment proférés lors des différentes crises, comme "Le drapeau ne sera jamais amené, l'appel à la prière ne cessera jamais " (Bayrak inmez, ezan dinmez), ${ }^{15}$. Le drapeau, symbole de la patrie, et l'appel à la prière, qui claque cinq fois par jour comme un drapeau sonore ${ }^{16}$, sont ainsi confondus en un même énoncé, et il y a dans ce slogan un raccourci de la synthèse turco-islamique, dont on peut observer maintes représentations photographiques dans le corpus rassemblé : inaugurations officielles lors desquelles les autorités font la prière sous des drapeaux turcs, et même cérémonies religieuses où des militaires, de haut grade (Türkiye, 24 décembre 1995) ou simples soldats (Ibid., 27 juillet 1996), en uniforme, prient ostensiblement ${ }^{17}$; photographies de diverses scènes de mosquées où le drapeau est présent, etc. La représentation du drapeau repose sur un code partagé par toute la population, notamment grâce à l'école ; ce code est en osmose avec d'autres systèmes de signes et il est susceptible d'évolution, comme le montre un examen même rapide de la presse de la décennie précédente, beaucoup moins imprégnée de ces images de drapeau : leur apparition est liée aux occurrences classiques, comme les fêtes nationales ou le protocole. En dehors des fêtes, la presse ne représentait des citoyens arborant le drapeau que dans certaines circonstances particulières : il s'agit souvent de photographies de Turcs vivant à l'étranger qui revendiquent ainsi leur appartenance, ou rappellent, de façon ostensible, leur fidélité à la patrie lointaine. Autant qu'on puisse en juger, il semble qu' une évolution se dessine également dès la fin de 1996. L'affaire dite de Susurluk, qui a révélé, grâce à un accident de la circulation (3 novembre 1996), les liens étroits existant entre la mafia, l'extrême-droite et l'Etat, a provoqué dans l'ensemble de la société un mouvement de rejet de certaines mises en scènes étatiques : le cercueil d'un grand truand décédé lors de cet accident, Abdullah Çatl_, ayant été revêtu du drapeau, une discrète réaction s'est ensuivie, et les occurrences de ce type se sont faites moins fréquentes. Il semble que certains cercles aient pris conscience qu'on 
était allé trop loin dans l'utilisation du drapeau ; celui-ci apparaît moins fréquemment dans les photographies d'arrestation ; la Sécurité d'Istanbul l'a remplacé par une grande photographie murale représentant les différents corps de police, de sorte que celle-ci n'est plus vraiment absente de la mise en scène, comme c'était le cas auparavant.

Toutefois, cette décrue n'affecte pas l'utilisation du drapeau par les citoyens euxmêmes ; le pavoisement reste très pratiqué, d'autant qu'il est question de le légaliser : en octobre 1996, un projet de loi a été proposé à l'Assemblée, qui permettrait aux institutions, aux commerçants et aux simples particuliers de pavoiser en permanence. Mais le plus frappant est l'appropriation croissante du drapeau par des forces politiques, comme s'il était devenu un emblême partisan. Le phénomène est sensible à l'extrême-droite puisqu'à l'automne 1996, les rues d'Istanbul ont été couvertes d'affiches-drapeaux, avec pour seule mention, très discrète, des "Foyers idéalistes d'Istanbul " (|stanbul Ülkü Ocaklar_), une organisation dépendant du parti d'extrêmedroite MHP ; un autre exemple était observable en février 1997, sur des affiches du MHP annonçant les meetings de son dirigeant, Alparslan Türkeò, sur un fond de cartedrapeau. Dans ces deux cas, le parti d'extrême-droite confisquait pour sa propagande l'emblême national, sans aucune apparition de ses propres emblêmes, l'image du loup gris ou la bannière rouge à trois croissants. Le phénomène avait commencé de toucher également le Refah, dont l'emblême était, avant son interdiction en janvier 1998, une parodie du drapeau turc (un croissant et un épi de blé parodiant eux-mêmes la faucille et le marteau communistes, blancs, sur fond rouge) : le 11 mai 1997, un grand meeting de soutien aux écoles religieuses, à Istanbul, était caractérisé par une utilisation massive du drapeau national. Ce cas peut être rapproché de certaines manifestations syndicales lors desquelles le drapeau est brandi par les manifestants; on observe la même pratique lors des manifestations de type écologiste, par exemples celles des habitants de Bergama protestant contre la pollution provoquée par la firme Eurogold dans leur région.

Le drapeau tend ainsi à devenir un symbole revendicatif, comme si les forces qui s'exprimaient à ces occasions estimaient représenter la vraie nation face à un État déconsidéré, surtout depuis l'affaire de Susurluk, par la corruption et la gabegie. Très paradoxalement, brandir le drapeau peut désormais signifier que l'on prend parti contre le gouvernement, ou même contre l'État. Le drapeau, dans ce cas, légitime la revendication et protège. Ce qui devrait être le symbole de l'unité nationale pourrait finalement devenir celui de la division, puisque des fractions de la nation cherchent à l'accaparer.

Il reste à s'interroger sur le phénomène observé en Turquie. Est-il banal, comme semble le suggérer l'existence de certains points communs avec d'autres sociétés, comme aux Etats-Unis (cérémonies patriotiques dans les écoles, large diffusion du drapeau et de son image) ou en Suisse, où l'on est également frappé par la très grande présence du drapeau national, et où il arrive même, comme en Turquie, que le drapeau soit inscrit dans le paysage, sur le flanc d'une montagne ? S'agit-il au contraire d'un phénomène propre à la Turquie, notamment dans ces manifestations si particulières que sont les photographies de funérailles et d'arrestations ? Des phénomènes semblables ont-ils, dans des sociétés si différentes, les mêmes causes et les mêmes significations ? Y a t-il quelque chose de commun à la Suisse, aux Etats-Unis et à la Turquie, qui inciterait Etat et population à rappeler sans cesse, visuellement, l'existence et la permanence de la nation? Pourquoi le Français, au contraire, réputé si chauvin, ne pavoise-t-il pas 
volontiers? Nous n'en sommes là qu'au début d'une réflexion sur le nationalisme et le patriotisme, sur ce que Billig appelle le nationalisme banal, et qui devra, en ce qui concerne la Turquie, être éclairé par une recherche sur le mot " nationalisme (milliyetçilik) " lui-même et sur ses différents usages.

\section{BIBLIOGRAPHIE}

\section{Sources}

Quotidiens dépouillés : Sabah, Türkiye, Hürriyet, Zaman, Cumhuriyet, Yeni Yüzyýl, Turkish Daily News ; dépouillement occasionnel : Radikal, Milliyet, AkÒam, Önce Vatan, Gözcü, Yeni Safak, Evrensel, Millî Gazete.

[Anonyme], 1990 et 1993, |lk Okul Türkçe Ders Kitabý1, Istanbul, Millî Egitim Basýmevi. [Anonyme], 1991, Liseler için Millî Güvenlik Bilgisi, 2-3, |stanbul, Millî Egitim Basýmevi, pp. 70-71.

Kantik B. et al., s.d., Renkli Atatürk Alfabesi, Istanbul, Serhat, 48 p.

Études

AbElès Marc, 1990, Anthropologie de l'État, Paris, Armand Colin, 183 p.

Barthes Roland, 1964, “ Rhétorique de l'image ”, Communication, 4, Paris, Seuil, pp. 40-52.

Billig Michael, 1997, Banal Nationalism, Londres, Sage, 200 p.

Copeaux Étienne, 1994, “ Manuels scolaires et géographie historique : le cas turc ", Hérodote, $n^{\circ}$ 74-75, pp. 196-240.

Copeaux Étienne, 1996, “Les mots de la mort dans les manuels d'histoire turcs ”, in Veinstein Gilles (dir.), Les Ottomans et la mort. Permanences et mutations, Leiden, New-York, Köln, Brill, pp. 303-324.

Copeaux Étienne, 1997, Espaces et temps de la nation turque. Analyse d'une historiographie nationaliste, 1931-1993, Paris, CNRS-Éditions, 369 p.

Copeaux Étienne, 1998, “ Bir Haritan_n Tarihi ” [Histoire d'une carte], Defter, 32, pp. 82-89 [en turc].

KALAYCI Hüseyin, 1998, "Bayrak, Milliyetsilik, Kutsallýk", Birikim, n 105-106, pp. 159-169.

Mauss-Copeaux Claire, 1996, “De l'Arabe aux Algériens : les images de l'autre dans les photographies du contingent ", in Blanchard P. et al., (dir.), L'autre et nous, "Scènes et types", Paris, Syros, ACHAC, pp. 247-252.

SEUFERT Günter, 1997, "The Sacred Aura of the Turkish Flag", New Perspectives on Turkey, n 16, pp. 53-61.

Veyne Paul, 1974, “L'histoire conceptualisante ”, in J. Le Goff et P. Nora (dir.), Faire de l'histoire. Nouveaux problèmes, Paris, Gallimard, 1974, pp. 62-92. 


\section{NOTES}

${ }^{1}$ Il est facile de percevoir une intention du photographe, dans ce cas, lorsque, par exemple, Necmettin Erbakan, Premier ministre islamiste, est cadré dans une attitude de prière avec un drapeau en arrière-plan (Milliyet, 29 juillet 1996) ou au contraire avec un portrait de Khomeiny (Sabah, 14 août 1996).

1. Est martyre toute personne décédée pour la patrie : soldats ou policiers lors d'affrontements ou simplement en service (même accidentellement) ; cf. Copeaux, 1996.

2. Dans le langage patriotique, on n'emploie jamais le mot "drapeau " pour désigner l'emblême d'un parti illégal, mais l'expression " morceau de chiffon (bez parçasý) ". 3. On peut y voir une référence implicite à la célèbre photographie des marines plantant le drapeau américain sur Iwo Jima (19 février 1945), d'autant plus qu'on trouve sur le marché des cartes postales patriotiques (éditions Efes Color, Istanbul), citant et reprenant cette scène avec des soldats turcs et un drapeau turc, dans la même attitude que les marines américains. On sait que la scène d'Iwo Jima a été reconstruite pour la prise de vue et non pas photographiée sur le vif.

4. Michael Billig $(1997,2)$ fait état d'une pratique semblable par The Sun au moment de la guerre des Malouines entre la Grande-Bretagne et l'Argentine (1982).

5. En turc, on distingue par les mots la couleur rouge du drapeau national (al) et celle du drapeau rouge de l'extrême-gauche (kýzýl).

6. Hürriyet, 20 juillet 1996 ; Ulutbatl_ Hasan est un héros de l'histoire populaire turque, passant pour le premier ottoman à avoir pénétré dans Constantinople et planté le drapeau impérial (mai 1453).

7. Du côté chypriote grec, on tient pour certain que l'auteur du coup de feu mortel est le ministre de l'Agriculture de la "République turque de Chypre du nord". Selon le rapport de l'ONU, le manifestant a été frappé de 5 balles.

8. Signalons cependant l'affaire Erbas : ce député islamiste de Van, qui a conduit une délégation dans un camp du PKK dans le nord de l'Irak pour négocier la libération de soldats turcs retenus en otage, a été photographié, à son insu, avec le drapeau kurde en arrière-plan. Certains clichés ont été composés de telle manière qu'il semble passer en revue un détachement de combattants kurdes. Les photos, plus que l'initiative d'Erbaò, ont fait scandale (30-31 août et jours suivants). Néanmoins, le député a été absous par la suite, puisqu'il a obtenu la libération des otages en décembre 1996, lors d'une nouvelle visite en Irak, au cours de laquelle il a pris soin de contrôler les prises de vue.

9. Cf respectivement, Sabah, 21 avril, 14 octobre 1996 ; Hürriyet, 26 juin 1996.

10. L'association rouge-sang-robe de fiancée-drapeau est explicite dans le célèbre poème d'Arif Nihat Asya, Bayrak. Le costume de cérémonie des petits garçons circoncis est souvent rouge et blanc lui aussi.

11. Un autre élément intéressant de cette caricature est que son auteur, Yurdagün, qui utilise fréquemment l'image cartographique de la Turquie dans ses dessins, situe la scène de part et d'autre de la mer Égée, alors que l'événement concerne théoriquement un Etat tiers et situé loin à l'est, la république de Chypre .

12. Cf aussi les déclarations d'I.H. Karadayý, chef d'Etat-major général, Hürriyet, 27 juin 1996.

13. Cette idéologie officieuse vise à une intégration des valeurs de l'islam dans le nationalisme turc ; elle définit la personnalité turque comme essentiellement musulmane (cf Copeaux, 1997). 
14. Dans une intention identique, il arrive que les mahya, ces inscriptions lumineuses déployées entre deux minarets d'une mosquée lors des fêtes, soient patriotiques et non plus religieuses. Ainsi, au moment de la fête de la République, le 29 octobre 1996, on pouvait lire entre les minarets de la Yeni Cami d'Istanbul : “ C'est l'unité qui est la base de la nation ", allusion claire au danger de sécession kurde.

15. Depuis la zone nord de Chypre, deux signes traversent la zone-tampon : l'immense drapeau nord-chypriote peint sur les flancs des monts Besparmak, visible depuis la partie sud; et l'appel à la prière de la mosquée Selimiye, audible à Nicosie-sud. 16. Voir également la presse du 15 janvier 1997 : lors d'une période de tension concernant Chypre, le général Karadayý, alors chef d'Etat-Major de l'armée turque, s'est fait photographier en attitude de prière dans le cimetière militaire de Bogaz, lors de sa visite à Chypre nord. Aucun journal, même à gauche, n'a cru bon d'attirer l'attention sur cette nouveauté dans le monde turc des signes. 